\title{
Nursing Homes of the Elderly in Asia: A comparison of Korea and Japan
}

\author{
Myung-Hee Jung* \\ Received: August 31, 2018. Revised: September 30, 2018. Accepted: October 15, 2018.
}

\section{Abstract}

Purpose - The purpose of this paper was to examine the views of nursing homes for the elderly in Korea and Japan.

Research design, data, and Methodology - This study aims to review the past researches on the social welfare and nursing homes for the elderly in Korea and Japan. A comprehensive view is offered with comparisons on the differences between the two countries regarding the dramatic increase of the elderly population.

Results - It could be seen that with the significant increase of the elderly populations, it is getting more difficult for families to take care of their elders. Thus, nursing homes offer an alternative to busy family members in the current times. Improvements are much needed in the nursing homes for the health of the elderly.

Conclusions - In order to offer better quality of life for the elderly in the nursing homes, more measures need to be taken to engage the elderly into activities. The depression that can be experienced when the elderly move to the nursing homes might be alleviated with new social circles and activities in the nursing homes, as well as human interactions with the staff

Keywords: Nursing Homes, Elderly, Korea, Japan.

JEL Classifications: J14, M52, L31, H53.

\section{Introduction}

In the age of globalization, the world is progressing at an exponential rate. With the increase in many exciting novel developments brought by the convergence of many different countries across the world, the living standards are also rising. Not only that, the fatality rate of many diseases that were thought to be incurable many years before are now solved in some cases with vaccine or medication. As such, this has also ushered in an era of the increase in aging societies.

With the increase of people living longer and better, societies are now confronted with the problem of a sudden surplus of the aging community. In the case of Japan and Korea, which are densely populated countries, the number of elderly people are growing. For the future of the elderly populations and their families, better nursing homes will need to be implemented. The health of the elderly will also be an important aspect, and the nursing homes should

\footnotetext{
* First Author. Professor, Department of Social Welfare, Jungwon University, Korea.

Tel: +82-43-830-8220. E-mail: jmhsubject@hanmail.net
}

implement various activities to encourage healthy habits in the elderly as well. Thus, this study aims to focus on the nursing homes and the situation of the elderly in Korea and Japan.

\section{Nursing homes and the elderly in Korea}

The proportion of the elderly in Korea is rising, and expected to increase to $14.3 \%$ by 2018 (Statistics Korea, 2018). Although the rise of the aging community indicates positive milestones to the overall increase in the wellness of the society, it is also creating many social issues with its alarming rate of increase. This significant rise in the aging community is bringing about new challenges to be tackled by the society, which includes economic problems, increased needs for healthcare, government funds, and changes in the position and social standing of the elderly in general. One of the major problems are the declining health of the elderly. Many are suffering from chronic diseases, which will require long-term treatment and inevitably lead to declining physical function, leading to strain on family members. The monetary factors are also a hurdle to be overcome, for it is taxing to 
provide care for chronic illnesses and they can be expensive on the long term.

In order to ease the burden handled by its citizens, the government has implemented the Long-Tern Care Insurance System (LTCIS) from July 2008 (Chang, 2013). This is in accord with a relief from the strenuous roles family caregivers have to give for their loved ones when they are transitioned to a nursing home instead (Gaugler, Pot, \& Zarit, 2007). But, the views from the actual elderly have to also be taken into consideration. More than the caretakers, the transition from a family to a nursing home is a huge leap in the environmental changes that will undoubtedly have an impact on the well-being of the elderly, both physically and psychologically.

This transition to a nursing home entails the adaptation of the elderly to new environments, life patterns, and social networks that can be trying for them in new situations (Lee, Woo, \& Mackenzie, 2002). It has been noted that the elderly experience bouts of stress from the adjustment and decreased contact with their familiars. Importantly, if the transition to a nursing home was an unwanted one, it has been revealed that there are higher mortality rates as well, leading to the negative impacts on the quality of life observed by the elderly with this sudden change (Heliker \& Scholler-Jaquish, 2006; Chao, Lan, Tso, Chung, Neim, \& Clark, 2008).

The transition of caring for the elderly from the families to the nursing homes have been gradual. The implementation of the Long-Term Care Insurance System (LTCIS) has really ignited the transition of the elderly care to the nursing homes. With the government's LTCIS, it has become assessible to everyone in utilizing nursing homes to take care of the elderly. The families have shifted their perspectives from thinking it mandatory to take care of the elderly members in their homes, to utilizing nursing home services (Shin, 2011).

Since the nursing home becomes the new place that the elderly associate their days with, it has become a place where the elderly from across different backgrounds and statuses all converge to one place and connect with each other (Lee et al., 2002). This makes it more imperative that the experiences the elderly have in the nursing homes are studied more in detail.

\section{Nursing homes and the elderly in Japan}

As is the case with Korea and most of the world population, the percentage of the elderly are increasing at an alarming rate. Japan was no exception in showing a significant increase of the elderly population. The Japanese families are similar to the Korean ones in that the elderly are usually taken care of by their children. However, in the modern society, it is getting more difficult to take care after the elderly, as the number of children has swindled in the recent years. But, it is conflicting for the Japanese people, as it is the tradition to take care of the elderly and to seek help outside of the family circle would be going against tradition or be seen as neglecting their elders (Oura, Washio, Wada, Arai, \& Mori, 2006).

With the increase in the elderly populations, Japan implemented its national long-term care insurance (LTCl) program in 2000. This was to differentiate between social and health care, and included services for adults that were aged 65 years or above regarding home care or residential services. Home care services are initiated under the LTCI program, where a care manager aids in the many daily chores of the elderly, including home help, day care, rehabilitation, and other needs (Nakanishi, Shimizu, Murai, \& Amaoka, 2015). This care was terminated when the elderly moved into nursing homes or other health related institutions. The Japanese government also introduced the concept of the unit care model, which is a large facility that is consisted of many small scale groups. This was a new concept at the structure of the nursing homes and the structure, but not on the care that was provided (Sawamura, Nakashima, \& Nakanishi, 2013).

Many studies have demonstrated the positive effects of social interactions of the elderly in improving their health. In a study with elderly patients with dementia, it was revealed that those in resident-style facilities had improved quality of life compared with those that did not engage in social interactions. It is important for the elderly to participate in recreational events, and regularly get visits from their children and relatives (Horiguchi, 1991).

\section{Improvements of nursing homes for the elderly}

As nursing homes are an institution, it is imperative that efforts are made to have the elderly feel more welcome and comfortable in the place they will be spending a majority of their lives. Thus, remodeling of the environments of the nursing homes are important, such as making it more of a cozy environment that is similar to a home than just an institution (Koren, 2010). This has been seen as an area that needed improvement, and several countries such as the Netherlands and Sweden, have tried to implement many aspects to make nursing homes feel like home.

One of the suggested methods for creating a home-like environment in the nursing homes has been to remodel the layout into one that was on a smaller scale. This can have many improved benefits for both the elderly and the workers, as smaller-scale homes indicate that the staff will have less to look after, and an overall decreased number of patients. Moreover, the important thing is that the elderly will also feel more at home from the size of the nursing homes 
and see that they are more cared for attentively, leading to a feeling of personal care (Rabig, 2009).

The smaller-scale nursing homes can arrange many benefits for the elderly, such as becoming a place where the elderly can form deep bonds and companionships with the other residents in the homes. One of the crucial aspects that contributed to the loneliness to the elderly in nursing homes was the lack of family but also a diminished sense of self-worth and loss of routine daily lives. Thus, with the smaller-scale nursing homes, the elderly were able to create their own group of companions for social purposes and also experience everyday, mundane activities (Verbeek, Zwakhalen, Rossum, Ambergen, Kempen, \& Hamers, 2010). This trend for smaller-scale nursing homes are demonstrating a consistent upward trends for other places internationally as well.

Interesting studies have been conducted on the quality of life for the elderly in Europe and the United States for small-scale nursing homes. Kane, Lum, Cutler, Degenholtz, and $\mathrm{Yu}$ (2007) have shown that in the United States, small-scale nursing homes improved the quality of life for the elderly than other conventional nursing homes. But, no significant founding could be uncovered from the small-scale homes of Belgium and the Netherlands in terms of depression of the elderly, or quality of life in countries such as Belgium and the Netherlands (De Rooij, 2012).

Nursing homes are not only an important factor in the health of the elderly, but a place where from the various social interactions, can help lead to a healthier mental state as well. The homelike environment will enable the elderly to be more engaged with their environment and interact more socially, creating a community that will have lasting effects on the relationships between the elderly people in the nursing homes. This is particularly imperative, as healthy social relationships can also lead to ultimately, a longer mortality rate that is always a key issue in the elderly populations and for their families as well. With increased positive energy in their lives, the elderly's health and mental state will improve, as well as the life-span and self-efficacy (Kiely \& Flacker, 2003).

A study conducted by Yoon et al. (2016) has demonstrated that the engagement of Korean elderly people in the nursing homes are relatively lower than those in other countries. It could be speculated that the nursing homes did not provide an abundance of activities from which to engage the elderly, and thus this in part contributed to the lack of connection and engagement in Korean elderly compared to other countries. An interesting perspective that should be taken into account, however, is the fact that since the introduction of the nursing homes were a relatively new concept in the Korean society, the foundations laid down are still new and in need of many improvements. Thus, as with the recent advances of nursing homes, the majority of nursing homes in Korea are focused on the clinical care of the elderly residents, as well as aiding with the daily chores and activities (Lee, Cho, \& Jang, 2009). Bang and Yun (2010) have also mentioned that more activities need to be incorporated into the daily lives of the elderly in the nursing homes.

Additionally, a beneficial aspect of the small-scale nursing homes can be the social connections that can be built between the elderly residents and the staff. As the number of staff are comparatively dwindled than conventional, larger homes, the elderly people will have a better chance to get to know the staff in a more intimate way and interact with them. The elderly residents are also more likely to engage in more activities and express themselves as they would feel comfortable in a close small-scale home (CohenMansfield, Marx, Thein, \& Dakheel-Ali, 2010). On a deeper analysis, this has implications for the mental well-being of the elderly residents as well and fewer depressive symptoms were shown (Cassie \& Cassie, 2012). One of the ways this can become a pleasant experience for the elderly as well is to include them in activities that makes their existence meaningful and worthy. Activities that the elderly can participate in will aid in renewed sense of purposes and the desire to live (Timonen \& O'Dwyer, 2009).

\section{Discussion}

This study has examined the many sides of nursing homes in the Asian countries, mainly Korea and Japan. The two countries have its own culture and differences in terms of nursing homes and the reactions to the elderly. The infrastructure and the policy systems from the governments were also varied. In relations to the behavior of the elderly ion nursing homes, Korean and Japanese elderly tended to have mixed feelings about the prospect of being in a nursing home.

In the cases where the elderly entering a nursing home is not due to their requests for such an arrangement, there are tendencies for negative feelings of desertion. As the era of which the elderly were raised are heavily focused on the Chinese cultural ways where the children are to look after the parents, it is a shock to most elderly when they are sent to nursing homes (Kim, 2010). From the perspective of the children, however, this is a scenario that is the most efficient, and not something that is considered to be particularly deserting of their elders. This can also be contributed to the fact that the age where the children of the elders were growing was a time for vast improvement in the economy and the convergence of the West culture as well, opening their eyes to industrialization and new social experiences. This, the viewpoint from the parent and the child can be different, and the reactions to the nursing houses differed as well.

One of the struggles the elderly face in the nursing homes is the detachment from family members. They had become accustomed to the family and the neighborhood, but 
to be ripped away from their comfort zone is a shock and sense of great emptiness for them. Thus, family visits to the nursing homes greatly aids in the lowering of the stress levels of elderly and the plays healing roles in their mental and physical state (Choi, Ransom, \& Wyllie, 2008). It is not an exaggeration to state that the elderly consider their family to become increasing valuable and important as time goes on (Jo, 2006). Despite all these barriers and negative feelings for the nursing homes, many elderly people were willing to stay and work it out if it meant helping their families and not creating more burden for them.

In order to ease the pressure from the families, but at the same time offer places for the elderly to be comfortable and create new social interactions among other things, many innovations are in progress. One of the ways was to create a small-scale nursing home, where the elderly residents might be able to feel more safe and relaxed than a conventional, large nursing house. The overall reactions to the small-scale nursing homes have been positive, with studies conducted in the United States and Europe. They have demonstrated lower levels of stress and improved quality of life (Kane et al., 2007).

In Korea, the activity labels of the elderly residents need to become more engaging, as compared to other countries, the level of participation of the elderly were lacking. With the relatively new incorporation of the nursing house systems into the society of Korea, there is a significant room for improvement, but are continually increasing in focusing on the other needs of the elderly to make nursing homes a more pleasant experience for the residents. In Japan as well, nursing homes should include more recreational activities and social opportunities for the elderly to engage themselves to the environment and live healthier lives.

Both Korea and Japan had implemented governmental policies regarding the matter of the increasing elderly populations in recent times. While Korea was more geared towards nursing home funding and support, Japan offered caregivers in residential houses until the elderly went to nursing homes. As supported by studies based on other Western countries, nursing homes needed to have a sense of comfort and small scale residences that felt like home (Koren, 2010; Rabig, 2009). Rather than large scale nursing homes, with numerous caregivers, the elderly were more comfortable with small scale nursing homes where they could get to know the residents and the nurses in the home. The social circles that are formed in the nursing homes and daily activities that the elderly participate in greatly helps curb potential depression and improve their overall health.

\section{Conclusions}

The current study has elucidated the nursing homes and the elderly in Korea and Japan. With the dramatic increase of the elderly in both countries, it is imperative that measures are taken to offer the best services to the elderly, but also for the families of the elderly people as well. With the current society, many family members have careers, and it is getting increasingly difficult to care for the elderly. With the busy lives of the modern society, it is inevitable that it will become increasingly hard to take care of the elderly and more institutions need to be implemented that are dedicated to the care of the elderly. This study has shown that Korean and Japanese governments have responded to the increasing number of elderly by implementing policies for nursing homes and its affiliated institutions. It is possible for the elderly to get depression at the abrupt change in the environment and the feeling that their family has abandoned them. But, it is important for the nursing homes to offer support for the elderly and create many recreational activities that will aid in the overall health of the elderly.

Many countries are facing problems with the sudden elderly population increase, but with the intervention of the government's policies regarding this matter and the improvement of the nursing homes will greatly increase the elderly's quality of life. Some of the limitations in this study was that this was a research based on two Asian countries of Korea and Japan. They hold similar traditions and are flourishing countries with a dense population of people and an increasing number of the elderly. It is suggested that for future research, more countries are compared in terms of the nursing homes and the situation of the elderly populations across the world. A study focused on the various kinds of nursing homes, as well as methods to help engage the elderly, will ne needed for the near future.

\section{References}

Bang, E. J., \& Yun, S. Y. (2010). Health needs of the elderly in long-term care facilities: Using RAI-MDS-FC. Journal of Korean Academy of Community Health Nursing, 21, 263-272.

Cassie, K. M., \& Cassie, W. E. (2012). Organizational and individual conditions associated with depressive symptoms among nursing home residents over time. The Gerontologist, 52, 812-821.

Chang, S. J. (2013). Lived experiences of nursing home residents in Korea. Asian Nursing Research, 72), 83-90.

Chao, S., Lan, Y., Tso, H., Chung, C., Neim, Y., \& Clark, M. (2008). Predictors of psychological adaptation among elderly residents in long-term care settings. Journal of Nursing Research, 16(2), 149-158

Choi, N., Ransom, S., \& Wyllie, R. (2008). Depression in older nursing home residents: the influence of nursing home environmental stressors, coping, and acceptance of group and individual therapy. Aging Mental Health, 12(5), 536-547 
Cohen-Mansfield, J., Marx, M. S., Thein, K., \& Dakheel-Ali, M. (2010). The impact of past and present preferences on stimulus engagement in nursing home residents with dementia. Aging \& Mental Health, 14, 67-73.

De Rooij, A. H. P. M., Luijkx, K. G., Schaafsma, J., Declercq, A. G., Emmerink, P. M. J., \& Schols, J. M. G. A. (2012). Quality of life of residents with dementia in traditional versus small-scale long-term care settings: A quasi-experimental study. International Journal of Nursing Studies, 49, 931-940.

Gaugler M., Pot, N., \& Zarit, S. (2007). Long-term adaptation to institutionalization in dementia caregivers. Gerontologist, 476), 730-740

Heliker, D., \& Scholler-Jaquish, A. (2006). Transition of new residents: Basing practice on residents' perspective. Journal of Gerontological Nursing, 32(9), 34-42

Horiguchi, J. (1991). A survey of the living condtions and psychological states of elderly peoplea dmitted to nursing homes in Japan, Acta psychiatrica scandinavica, 83(5), 338-341.

Jo, S.N. (2006). Challenge of the aging society and familial support for the elderly. Korean Journal of Population Studies, 29(3).

Kane, R. A., Lum, T. Y., Cutler, L. J., Degenholtz, H. B., \& Yu, T.-C. (2007). Resident outcomes in small-house nursing homes: a longitudinal evaluation of the initial green house program. Journal of the American Geriatrics Society, 55, 832-839.

Kiely, D. K., \& Flacker, J. M. (2003). The protective effect of social engagement on 1-year mortality in a long-stay nursing home population. Journal of Clinical Epidemiology, 56, 472-478.

Kim, Y. B. (2010). Supporting the old and filial piety. Seoul, Korea: Sohwa Press.

Koren, M. J. (2010). Person-centered care for nursing home residents: The culture-change movement. Health Affairs, 29, 312-317.

Lee, T. W., Cho, S. Y., \& Jang, Y. K. (2009). Predictors of nursing service need for nursing homes residents. Journal of Korean Academy of Nursing, 39, 95-106.
Lee, D., Woo, J., Mackenzie, A. (2002). A review of older people's experiences with residential care placement. Journal of Advanced Nursing, 37(1), 19-27

Nakanishi, M., Nakashima, T., \& Sawamura, K. (2013). Quality of life of residents with dementia in a group-living situation: An approach to creating small, homelike environments in traditional nursing homes in Japan, Japanese Journal of Public Health, 591), 3-10.

Nakanishi, M., Shimizu, S., Murai, T., \& Amaoka, A. (2015). "Ageing in place" policy in Japan: Association between the development of an integrated community care system and the number of nursing home placements under the public long-term care insurance program among the municipal governments. Ageing Int, 40, 248-261.

Oura, A., Washio, M., Wada, J., Arai, Y., \& Mori, M. (2006). Factors related to institutionalization among the frail elderly with home-visiting nursing service in Japan, Gerontology, 52, 66-68.

Rabig, J. (2009). Home again: Small houses for individuals with cognitive impairment. Journal of Gerontological Nursing, 35, 10-15.

Sawamura, K., Nakashima, T., \& Nakanishi, M. (2013). Provision of individualized care and built environment of nursing homes in Japan, Archives of gerontology and geriatrics, 56(3), 416-424.

Shin, 2011, K.A. Shin, Defamilization of elderly care and the experiences of the aged, Korean Journal of Sociology, 45(4), 64-96

Timonen, V. (2009). No Title Here Social Work in Health Care, 48(6), 597-613

Verbeek, H., Zwakhalen, S. M. G., Rossum, E., Ambergen, T., Kempen, G. I. J. M., \& Hamers, J. P. H. (2010). Small-scale, homelike facilities versus regular psychogeriatric nursing home wards: A cross-sectional study into residents' characteristics. BMC Health Services Research, 10, 30.

Yoon, J. Y., Kim, H., Jung, Y., \& Ha, J. (2016). Impact of the nursing home scale on residents' social engagement in South Korea. International Psychogeriatrics, 28(12), 1965-1973 